\title{
Programming the projections
}

The children of mothers with impaired metabolic homeostasis, as occurs in obesity, have an increased risk of metabolic disorders. The mechanisms underlying this 'metabolic programming' are poorly understood but are thought to involve changes in the offspring's hypothalamic circuits - which regulate energy and glucose homeostasis. A study in mice now shows that excessive maternal calorie intake during lactation alters hypothalamic axon projections in offspring, partly by increasing insulin signalling.

In murine hypothalamus development, neuronal number is established in utero, but functional neuronal networks are formed postnatally. To ascertain which of these development phases might be sensitive to altered maternal metabolism, the authors fed female mice a high-fat diet (HFD) before they gave birth and/or during lactation and then assessed their offspring for metabolic defects. Only the offspring of females fed a HFD during lactation exhibited an obese phenotype, suggesting that altered maternal metabolic homeostasis affects postnatal neuronal network formation.

The arcuate nucleus of the hypothalamus $(\mathrm{ARH})$ contains two functionally opposed neuronal populations that have key roles in regulating metabolism: anorexigenic pro-opiomelanocortin (POMC)expressing and orexigenic agoutirelated protein (AGRP)-expressing neurons. Both neuronal populations exert their effects via projections to other hypothalamic areas, including the pre-autonomic paraventricular nucleus of the hypothalamus (PVH).

The authors sought to identify abnormalities in this circuitry in the male offspring of females fed on a HFD during lactation by assessing immunoreactivity for AGRP and a-melanocyte-stimulating hormone ( $\alpha$-MSH) - the processed, active form of POMC. Strikingly, these mice exhibited a marked decrease in AGRP- and $\alpha-\mathrm{MSH}$-expressing axons in the PVH and other ARH neuron target sites, indicating that defects in hypothalamic axon formation contribute to metabolic programming in mice.

Female mice fed on a HFD had increased levels of glucose and insulin in their milk, and their offspring had abnormally high blood insulin levels at 3 weeks of age. Thus, the authors examined whether abnormal insulin signalling contributes to the development of hypothalamic circuit defects. They generated litters comprising wildtype offspring and mice in which the insulin receptor was inactivated in POMC-expressing neurons. The mothers of these mice were then fed a HFD during lactation.

Although offspring in both groups showed metabolic defects, only wild-type offspring showed glucose intolerance when tested at 15 weeks of age. Moreover, insulin receptor inactivation specifically prevented the loss of $\alpha$-MSHexpressing axons in a subcompartment of the PVH. Thus, increased insulin signalling induces abnormal outgrowth of a subpopulation of POMC-expressing neurons and underlies maternal HFD-induced hyperinsulinaemia.

Together, these data show that, in mice, abnormal maternal metabolic homeostasis metabolically programmes offspring by altering hypothalamic circuits. Human hypothalamic circuit development occurs in the third trimester of pregnancy, suggesting that similar axon projection changes occur prenatally in humans during metabolic programming.

Darran Yates

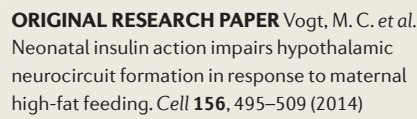

C6

Together, these data show that, in mice, abnormal maternal metabolic homeostasis metabolically programmes offspring by altering hypothalamic circuits.

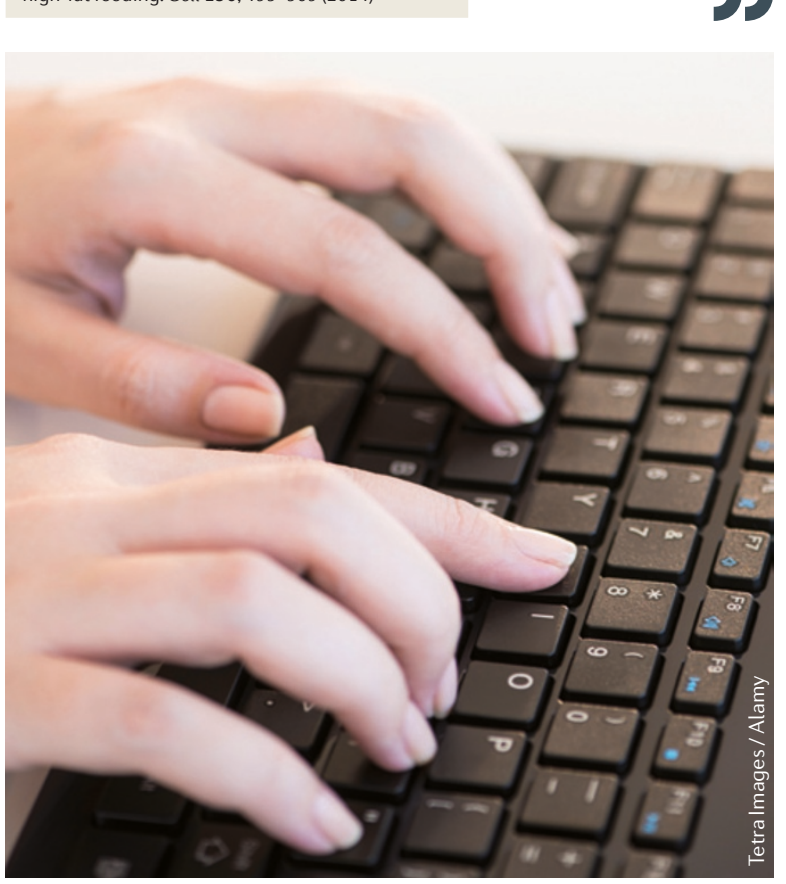

